\title{
Supervised and induced sputum among patients with smear-negative pulmonary tuberculosis
}

\author{
K.C. Chang*, C.C. Leung*, W.W. Yew ${ }^{\#}$ and C.M. Tam*
}

ABSTRACT: Sputum culture is essential for monitoring drug resistance. Although sputum induction may optimise culture yield, better selection criteria and simpler algorithms are needed for countries with intermediate tuberculosis burdens.

From a cohort of 660 patients who registered for antituberculosis treatment in a government chest clinic from May 21, 2005 to February 28, 2007, 187 patients with pulmonary disease and a negative smear in two unsupervised sputum specimens were enrolled prospectively for collection of one specimen each of supervised and induced sputum in succession.

Among enrolled patients, induced sputum significantly improved ease of expectoration on a subjective five-point scale. Among 78 patients with culture-proven pulmonary tuberculosis, analysis of matched sputum culture results showed that: 1) induced sputum outperformed supervised sputum; 2) the second unsupervised sputum was significantly inferior to the first and redundant in the presence of the others; 3) adding one specimen each of supervised and induced sputum to two unsupervised specimens increased culture yield significantly; and 4) patients with either extent of disease less than right upper lobe or no respiratory symptoms were more likely to benefit.

In summary, it may be practical to collect a sample of unsupervised, supervised and induced sputum for smear-negative patients with extent of disease less than the right upper lobe, especially when respiratory symptoms are absent.

KEYWORDS: Culture, diagnosis, induced sputum, supervised sputum, tuberculosis

putum culture, useful for confirming pulmonary tuberculosis (TB), is essential for drug susceptibility testing [1], which is becoming increasingly important in an era of increasing drug-resistance [2], multidrug-resistant TB [3] and extensively drug-resistant TB [4].

Introduced $>40$ yrs ago [5-9], sputum induction has been advocated for improving diagnostic yield in patients with difficulty in expectorating $[10,11]$. Its efficacy is probably comparable with bronchoalveolar lavage [12-15]. It is uncertain whether sputum induction may owe its efficacy to supervision. Although several studies [12, 14, 16 , 17] showed sputum induction was costsaving, it may be difficult for countries with intermediate TB burdens to spend $30 \mathrm{~min}$ on sputum induction indiscriminately and virtually impossible to spend up to $2 \mathrm{~h}$ [18] to induce sputum three times [14, 18]. Better selection criteria and simpler algorithms are needed.

Hong Kong (China) has been classified as having an intermediate TB burden [19]. In 2005, TB notifications and rate were $\sim 6,000$ cases and 90 cases per 100,000 population, respectively, [20]. Approximately one-third of pulmonary TB cases were smear-positive, whereas two-thirds were culture-proven [20]. Despite a common practice of collecting up to four sputum specimens before treatment, the prevalence of culture-positive cases among smear-negative patients was only $52 \%$ [20]. Although sputum induction might enhance diagnostic yield, it has never been evaluated in Hong Kong.

In view of a lack of systematic studies of supervised and induced sputum for countries with intermediate TB burden, a prospective study was conducted among patients with smear-negative pulmonary TB disease to identify an optimal algorithm for sputum collection.

\section{METHODS}

From May 21, 2005 to February 28, 2007, patients suspected of pulmonary TB disease with negative
AFFILIATIONS

*Tuberculosis and Chest Service, Centre for Health Protection, Departrment of Health, and

"Tuberculosis and Chest Unit, Grantham Hospital, Hong Kong, China.

CORRESPONDENCE

K.C. Chang

Wanchai Chest Clinic

1st Floor

Wanchai Polyclinic

99

Kennedy Road

Wanchai

Hong Kong

China

Fax: 85228346627

E-mail: kc_chang@dh.gov.hk

Received:

September 172007

Accepted after revision:

December 252007

STATEMENT OF INTEREST

None declared. 


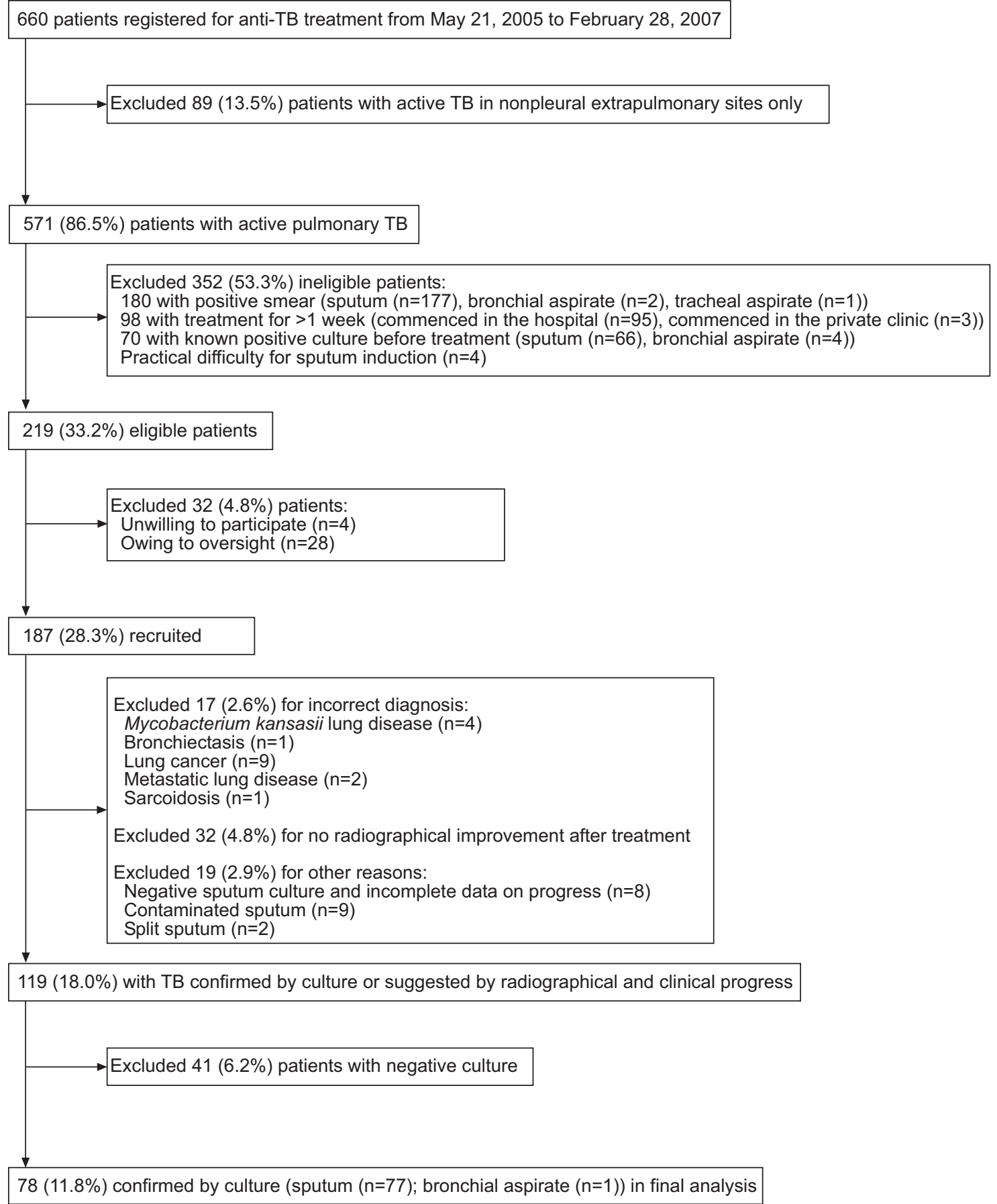

FIGURE 1. Flow diagram showing enrolment of patients from a cohort of 660 patients. TB: tuberculosis.

acid-fast bacilli smear in two unsupervised sputum specimens were enrolled prospectively from a government chest clinic (Yaumatei Chest Clinic, Hong Kong) for collecting one specimen each of supervised and induced sputum in immediate succession. Thus, each participant had four sputum specimens. TB was diagnosed based upon clinical and radiographical findings coupled with absence of response to empirical antibiotics. Public health nurses instructed all patients on how to cough and produce sputum [21]. Sputum specimens were sent to the Supranational TB Reference Laboratory in
Hong Kong for processing. All specimens were examined macroscopically according to a published guide [21]. Only empty bottles and specimens that were dried up or considered insufficient were rejected.

Some specimens were excluded from enrolment due to: TB occurring only in nonpleural extrapulmonary sites; positive smear or culture in any respiratory specimen; anti-TB treatment received for $>1$ week before sputum induction; practical difficulty in inducing sputum; or lack of consent. 
Before or within 1 week after commencement of treatment, supervised expectoration followed immediately by sputum induction was performed inside a transparent local exhaust ventilation booth, which was built in accordance with published guidelines [22, 23]. Patients fasted for $\geqslant 2 \mathrm{~h}$ beforehand. Nebulised hypertonic saline (5.85\% (weight/volume)) delivered with a high-flow jet nebuliser for $20 \mathrm{~min}$ was used to induce sputum. Blood pressure and peak expiratory flow rate (PEFR) were measured before and after sputum induction. The patients' ease of sputum production was measured before and after sputum induction on a subjective five-point scale, comprising the following items ranking 1-5, respectively: "no sputum", "difficult", "okay after effort", "easy" and "effortless". Wilcoxon signed rank test was used to compare ranks obtained before and after sputum induction.

Paired culture results obtained by alternative sputum collection methods were compared among patients with cultureproven pulmonary TB. Factors that might associate with culture gain from supervised and induced sputum relative to two unsupervised specimens were evaluated by univariate and multivariable analyses. For multivariable analysis, factors with $p$-values $<0.05$ by univariate analysis were included by default, whereas those with $\mathrm{p}$-values $<0.2$ were included by forward stepwise selection using p-values of 0.05 and 0.10 as cut-offs for entry and removal, respectively.

Approval and informed consent were obtained from the institutional review board and each participant, respectively.

\section{RESULTS}

Figure 1 shows enrolment from a cohort of 660 patients who registered for anti-TB treatment from May 21, 2005 to February 28,2007 . There was no significant difference between 187 enrolled patients and 32 eligible patients that were excluded due to oversight or unwillingness to participate.

Among 187 enrolled patients, sputum induction increased ease of expectoration significantly as compared with supervised collection on a subjective five-point scale (median score 3 versus 2 , respectively; $\mathrm{p}<0.001$ ). Sputum induction was aborted in two $(1.1 \%)$ patients owing to transient numbness or dizziness with chest pain, which disappeared spontaneously. A total of nine $(4.8 \%)$ patients had minor adverse events that did not require termination of sputum induction or treatment: nausea $(n=1)$, chest pain $(n=1)$, dizziness $(n=2)$, numbness $(n=2)$, epigastric discomfort $(n=1)$ and transient asymptomatic fall in PEFR $(n=2)$

Table 1 shows comparison of paired culture results obtained by alternative sputum collection methods among 78 patients with culture-proven pulmonary TB. The second unsupervised sputum was significantly inferior to the first. Induced sputum significantly outperformed supervised sputum. Adding one specimen each of supervised and induced sputum to two unsupervised specimens increased culture yield significantly.

Table 2 shows the results of univariate analysis of factors that may associate with culture gain from supervised and induced sputum, relative to two unsupervised specimens among the 78 patients with culture-proven pulmonary TB. Only extent of disease less than right upper lobe and lack of respiratory symptoms were significantly associated with culture gain in both univariate and multiple logistic regression analyses; the adjusted odds ratios (95\% confidence interval) were 7.2 (1.928.0) and 3.0 (1.0-8.9), respectively.

Table 3 shows the sensitivity of smear and culture in different sputum collection methods stratified by disease extent and respiratory symptoms. By including a specimen of supervised and induced sputum to two unsupervised specimens, the sensitivity for culture in descending order of magnitude was: from 26 to $95 \%$ when the extent of disease was less than the right upper lobe with no respiratory symptoms; from 50 to $100 \%$ when the extent of disease was less than right upper lobe with respiratory symptoms; and from 86 to $100 \%$ when the extent of disease exceeded right upper lobe. The corresponding changes for sputum smear, which occurred in the reverse direction, were $0-0 \%, 0-8 \%$ and $0-19 \%$, respectively. The second unsupervised specimen was redundant in the presence of the other specimens.

\begin{tabular}{|c|c|c|c|c|c|c|}
\hline Method 1 & Method 2 & $\begin{array}{c}\text { Both } \\
\text { negative }\end{array}$ & $\begin{array}{l}\text { Both } \\
\text { positive }\end{array}$ & $\begin{array}{l}\text { Positive in } \\
\text { method } 1 \text { only }\end{array}$ & $\begin{array}{l}\text { Positive in } \\
\text { method } 2 \text { only }\end{array}$ & $\begin{array}{l}\text { \% difference between } \\
\text { paired proportions } \\
(95 \% \mathrm{Cl})\end{array}$ \\
\hline First unsupervised sputum & Second unsupervised sputum & 36 & 18 & $18(23.1)$ & $6(7.7)$ & $15.4(3.1-27.7)$ \\
\hline $\begin{array}{l}\text { Two unsupervised and one } \\
\text { supervised sputa }\end{array}$ & Two unsupervised sputa & 18 & 42 & $18(23.1)$ & $0(0)$ & $23.1(12.4-33.7)$ \\
\hline $\begin{array}{l}\text { Two unsupervised, one supervised } \\
\text { and one induced sputa }\end{array}$ & $\begin{array}{l}\text { Two unsupervised and one } \\
\text { supervised sputa }\end{array}$ & 1 & 60 & $17(21.8)$ & $0(0)$ & $21.8(11.4-32.2)$ \\
\hline $\begin{array}{l}\text { Two unsupervised, one supervised } \\
\text { and one induced sputa }\end{array}$ & Two unsupervised sputa & 1 & 42 & $35(44.9)$ & $0(0)$ & $44.9(30.0-59.7)$ \\
\hline
\end{tabular}




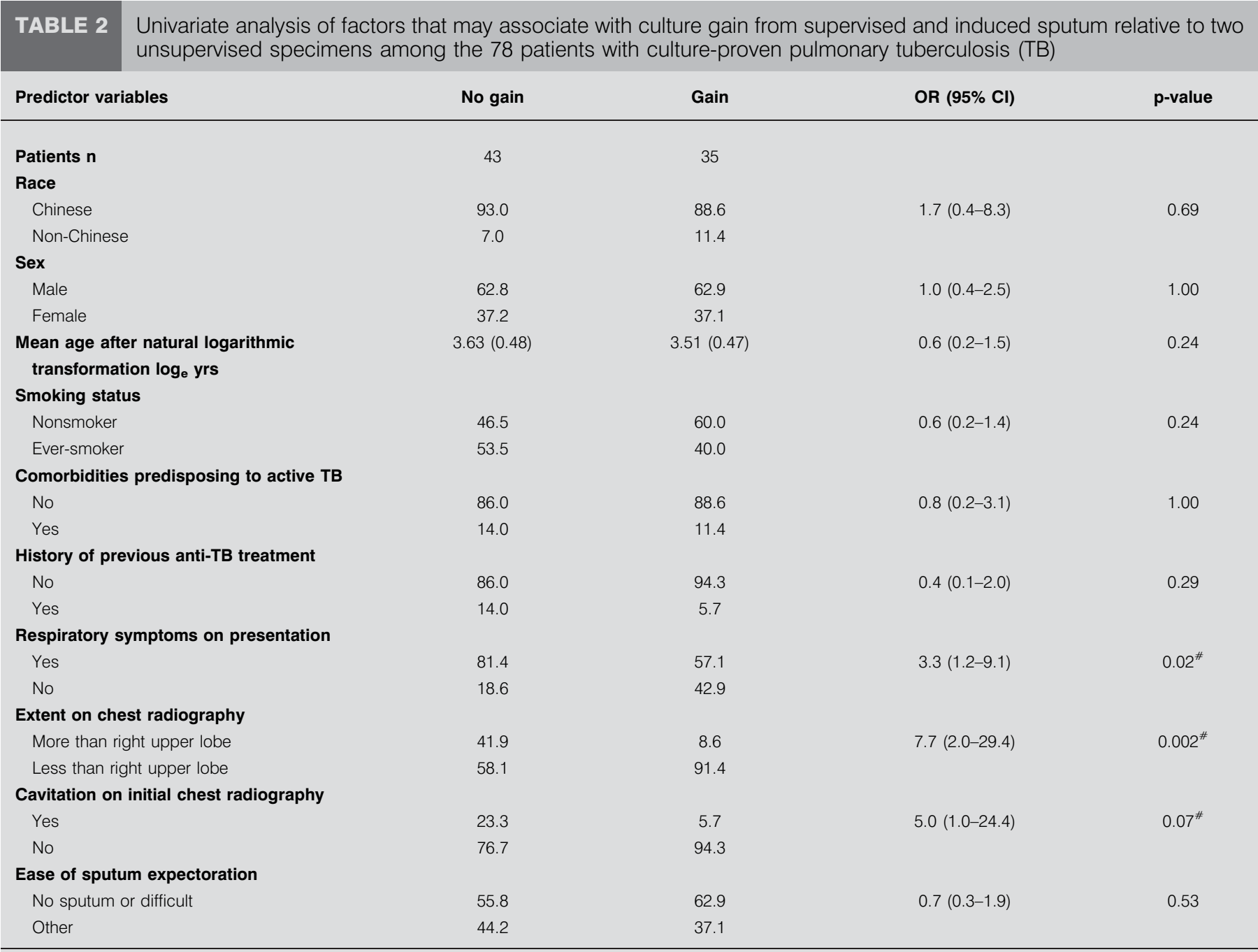

Data are presented as \%, unless otherwise stated. The first subgroup is the reference group for interpreting odds ratios. Natural logarithmic transformation was applied to normalise the distribution of age, which was positively skewed. OR: odds ratio; Cl: confidence interval. ${ }^{*}$ : factors with a p-value $<0.2$ were further analysed in a logistic risk model of sputum culture gain by including factors with a $p$-value $<0.05$ by default and selecting the rest by forward stepwise regression using $p$-values of 0.05 and 0.10 as cut-offs for entry and removal, respectively. Compared with counterparts, extent of disease less than right upper lobe and lack of respiratory symptoms on presentation were significantly associated with sputum culture gain, adjusted OR $(95 \% \mathrm{Cl})$ being $7.2(1.9-28.0)$ and $3.0(1.0-8.9)$, respectively. Thus, smear-negative patients with extent of disease less than right upper lobe or no respiratory symptoms were more likely to benefit from supervised expectoration and sputum induction.

\section{DISCUSSION}

The present study has provided more evidence supporting the use of sputum induction in smear-negative pulmonary ТВ disease. First, induced sputum significantly outperformed supervised sputum. It is probable that hypertonic saline works by improving sputum transportability, enhancing mucociliary clearance and inducing cough [24, 25]. Secondly, by adding one specimen each of supervised and induced sputum to two unsupervised specimens the culture yield was significantly increased. Although the reference group comprised two unsupervised specimens instead of the current standard of three, the difference could be negligible given the significantly inferior performance of the second unsupervised specimen relative to the first. The latter is consistent with previous studies [26]. It is probably unnecessary to request more than one unsupervised specimen if both supervised expectoration and sputum induction are contemplated.

The present study may also help to refine selection criteria for sputum induction. Smear-negative patients with either extent of disease less than right upper lobe or no respiratory symptoms were more likely to benefit from supervised expectoration and sputum induction. This is consistent with previous findings supporting the role of sputum induction for tuberculous pleural effusion [27], in which lung disease is often minimal.

In accordance with a previous study [28], which showed no significant difference between induced sputum and routineexpectorated sputum in establishing a diagnosis of smearpositive TB disease, the present study showed that only $8 \%$ of symptomatic patients with smear-negative disease of extent 


\begin{tabular}{|c|c|c|c|c|c|c|c|c|c|}
\hline TABLE 3 & $\begin{array}{l}\text { Sensitiv } \\
\text { proven }\end{array}$ & $\begin{array}{l}\text { f sme } \\
\text { nonary }\end{array}$ & $\begin{array}{l}\text { culture } \\
\text { culosis }\end{array}$ & rent si & collectic & hods ar & a cohort & patien & culture- \\
\hline \multirow{2}{*}{\multicolumn{2}{|c|}{$\begin{array}{l}\text { Sputum collection } \\
\text { methods }\end{array}$}} & \multicolumn{6}{|c|}{ Patients subgroups } & \multirow{2}{*}{\multicolumn{2}{|c|}{ All $^{f}$}} \\
\hline & & \multicolumn{2}{|c|}{$\begin{array}{l}\text { Without respiratory } \\
\text { symptoms }{ }^{\#}\end{array}$} & \multicolumn{2}{|c|}{$\begin{array}{l}\text { With respiratory } \\
\text { symptoms }\end{array}$} & \multicolumn{2}{|c|}{ Extent $>$ right upper lobe $^{+}$} & & \\
\hline \multirow{2}{*}{\multicolumn{2}{|c|}{$\begin{array}{l}\text { Two unsupervised sputa } \\
\text { Two unsupervised and } \\
\text { one supervised sputa }\end{array}$}} & $0(0)$ & $5(26)$ & $0(0)$ & $19(50)$ & $0(0)$ & $18(86)$ & $0(0)$ & $42(54)$ \\
\hline & & $0(0)$ & $11(58)$ & $3(8)$ & $30(79)$ & $3(14)$ & $19(90)$ & $6(8)$ & $60(77)$ \\
\hline \multicolumn{2}{|c|}{$\begin{array}{l}\text { Two unsupervised and } \\
\text { one induced sputa }\end{array}$} & $0(0)$ & $17(89)$ & $3(8)$ & $34(89)$ & 3 (14) & $21(100)$ & $6(8)$ & 72 (92) \\
\hline \multicolumn{2}{|c|}{$\begin{array}{l}\text { Two unsupervised, one } \\
\text { supervised and one } \\
\text { induced sputa }\end{array}$} & $0(0)$ & $18(95)$ & $3(8)$ & $38(100)$ & $4(19)$ & $21(100)$ & $7(9)$ & 77 (99) \\
\hline
\end{tabular}

less than right upper lobe would become smear-positive after collecting additional supervised and induced sputum. When the initial sputum smear is negative, additional supervised and induced sputum would mainly enhance culture yield and drug susceptibility testing without establishing a rapid bacteriological diagnosis. Treatment would still need to be initiated on clinical and radiographical grounds.

Inaccessibility to data on disease extent and respiratory symptoms in the published literature may make it difficult to compare supervised and induced sputum with multiple sputum induction. Reorganisation of data from a recent study [15] showed that two additional induced sputum specimens increased culture yield of two spontaneous sputum specimens from 74 to $100 \%$ among 27 patients with culture-proven TB. This order of gain may be less than that for extent of disease less than right upper lobe but comparable with that for more extensive disease.

In countries of intermediate TB burden, a triplet of unsupervised, supervised and induced sputum may be more practicable than three induced sputum specimens. Thus, the current authors recommend the following algorithm. For lesions less than right upper lobe, check unsupervised sputum once, especially when respiratory symptoms are absent, and prescribe antibiotics empirically [29]. If smear-negative TB disease is suspected, collect one specimen each of supervised and induced sputum in immediate succession inside a local exhaust ventilation device $[22,23]$. Costs may be outweighed by savings on laboratory consumables, manpower and bronchoscopy.

In summary, the present study has reaffirmed the role of sputum induction, refined its selection criteria and provided a practicable algorithm for optimising culture yield in smearnegative pulmonary tuberculosis disease.

\section{ACKNOWLEDGEMENTS}

The authors would like to thank C. Chan (Tuberculosis \& Chest Service, Hong Kong, China), our senior nursing officer, and all colleagues of Yaumatei Chest Clinic for their dedicated and professional support in the study. The authors would additionally like to thank T.Y. Lau, D.H.C. Lam, K.Y.K. Lau, W.L. Law, F. Cheung, S.P. Wong, A. Fung and S. Sin (Tuberculosis \& Chest Service). Finally, the authors would also like to thank C.K. Chan (Tuberculosis \& Chest Service) for conducting a small pilot study of sputum induction in another chest clinic (Wanchai Chest Clinic, Hong Kong) before this study was conceived.

\section{REFERENCES}

1 Van Deun A. What is the role of mycobacterial culture in diagnosis and case definition? In: Frieden T, ed. Toman's Tuberculosis Case Detection, Treatment, and Monitoring: Questions and Answers. 2nd Edn. Geneva, World Health Organization, 2004; pp. 35-43.

2 Bell D, Leckie V, McKendrick M. The role of induced sputum in the diagnosis of pulmonary tuberculosis. J Infect 2003; 47: 317-321.

3 World Health Organization. Guidelines for the Programmatic Management of Drug-resistant Tuberculosis. WHO/HTM/ TB/2006.361. Geneva, World Health Organization, 2006.

4 Migliori GB, Loddenkemper R, Blasi F, Raviglione MC. 125 years after Robert Koch's discovery of the tubercle bacillus: the new XDR-TB threat. Is "science" enough to tackle the epidemic? Eur Respir J 2007; 29: 423-427.

5 Hensler NM, Spivey CG Jr, Dees TM. The use of hypertonic aerosol in production of sputum for diagnosis of tuberculosis. Comparison with gastric specimens. Dis Chest 1961; 40: 639-642.

6 Lillehei JP. Sputum induction with heated aerosol inhalations for the diagnosis of tuberculosis. Am Rev Respir Dis 1961; 84: 276-278.

7 Elliott RC, Reichel J. The efficacy of sputum specimens obtained by nebulization versus gastric aspirates in the bacteriologic diagnosis of pulmonary tuberculosis. A comparative study. Am Rev Respir Dis 1963; 88: 223-227. 
8 Schwartz I, Small MJ. Preliminary studies in the use of superheated saline nebulization in the bacteriologic diagnosis of pulmonary tuberculosis. Am Rev Respir Dis 1961; 84: 279-280.

9 Ledwith JW. The induced sputum technique for collection of tuberculosis cultures: effectiveness compared to gastric aspiration. Tuberculol Thorac Dis 1965; 23: 12-17.

10 Al Zahrani K, Al Jahdali H, Poirier L, René P, Menzies D. Yield of smear, culture and amplification tests from repeated sputum induction for the diagnosis of pulmonary tuberculosis. Int J Tuberc Lung Dis 2001; 5: 855-860.

11 Zar HJ, Hanslo D, Apolles P, Swingler G, Hussey G. Induced sputum versus gastric lavage for microbiological confirmation of pulmonary tuberculosis in infants and young children: a prospective study. Lancet 2005; 365: 130-134.

12 Anderson C, Inhaber N, Menzies D. Comparison of sputum induction with fiber-optic bronchoscopy in the diagnosis of tuberculosis. Am J Respir Crit Care Med 1995; 152: 1570-1574.

13 Conde MB, Soares SL, Mello FC, et al. Comparison of sputum induction with fiberoptic bronchoscopy in the diagnosis of tuberculosis: experience at an acquired immune deficiency syndrome reference center in Rio de Janeiro, Brazil. Am J Respir Crit Care Med 2000; 162: 2238-2240.

14 McWilliams T, Wells AU, Harrison AC, Lindstrom S, Cameron RJ, Foskin E. Induced sputum and bronchoscopy in the diagnosis of pulmonary tuberculosis. Thorax 2002; 57: 1010-1014.

15 Schoch OD, Rieder P, Tueller C, et al. Diagnostic yield of sputum, induced sputum, and bronchoscopy after radiologic tuberculosis screening. Am J Respir Crit Care Med 2007; 175: 80-86.

16 Li LM, Bai LQ, Yang HL, et al. Sputum induction to improve the diagnostic yield in patients with suspected pulmonary tuberculosis. Int J Tuberc Lung Dis 1999; 3: 1137-1139.

17 Harmon JC, Roche JM. Development of a research-based protocol to rule out tuberculosis by means of continuous quality improvement techniques. Am J Infect Control 1995; 23: 329-336.

18 Brown M, Varia H, Bassett P, Davidson RN, Wall R, Pasvol G. Prospective study of sputum induction, gastric washing, and bronchoalveolar lavage for the diagnosis of pulmonary tuberculosis in patients who are unable to expectorate. Clin Infect Dis 2007; 44: 1415-1420.

19 World Health Organization, Regional Office for the Western Pacific. Fighting TB - Forging Ahead: Overview of the Stop TB Special Project in the Western Pacific Region. Geneva, World Health Organization, 2002.

20 Hong Kong Tuberculosis and Chest Service. Annual Report. Hong Kong, Department of Health of the Government of the Hong Kong Special Administrative Region, 2005.

21 International Union Against Tuberculosis and Lung Disease (IUATL). Technical guide - Sputum Examination for Tuberculosis by Direct Microscopy in Low Income Countries. 5th Edn. Paris, IUATL, 2000.

22 Guidelines for preventing the transmission of Mycobacterium tuberculosis in health-care facilities, 1994. Centers for Disease Control and Prevention. MMWR Recomm Rep 1994; 43: 1-132.

23 Jensen PA, Lambert LA, Iademarco MF, Ridzon R, Centers for Disease Control and Prevention. Guidelines for preventing the transmission of Mycobacterium tuberculosis in health-care settings, 2005. MMWR Recomm Rep 2005; 54: 1-141.

24 Scheicher ME, Terra Filho J, Vianna EO. Sputum induction: review of literature and proposal for a protocol. Sao Paulo Med J 2003; 121: 213-219.

25 Wills PJ, Hall RL, Chan W, Cole PJ. Sodium chloride increases the ciliary transportability of cystic fibrosis and bronchiectasis sputum on the mucus-depleted bovine trachea. J Clin Invest 1997; 99: 9-13.

26 Harries A. What is the additional yield from repeated sputum examinations by smear microscopy and culture? In: Frieden T, ed. Toman's Tuberculosis Case Detection, Treatment, and Monitoring: Questions and Answers. 2nd Edn. Geneva, World Health Organization, 2004; pp. 46-50.

27 Conde MB, Loivos AC, Rezende VM, et al. Yield of sputum induction in the diagnosis of pleural tuberculosis. Am J Respir Crit Care Med 2003; 167: 723-725.

28 Merrick ST, Sepkowitz KA, Walsh J, Damson L, McKinley P, Jacobs JL. Comparison of induced versus expectorated sputum for diagnosis of pulmonary tuberculosis by acid-fast smear. Am J Infect Control 1997; 25: 463-466.

29 Tuberculosis Coalition for Technical Assistance (TCBTA). International Standards for Tuberculosis Care. The Hague, TBCTA, 2006. 